\title{
Inner Finger Formation During Droplet Impact on Liquid Films
}

\author{
Nuri Erdem Ersoy ${ }^{1}$, David LS Hung ${ }^{* 1}$, Morteza Eslamian ${ }^{1,2}$ \\ ${ }^{1}$ University of Michigan-Shanghai Jiao Tong University Joint Institute, Shanghai, China \\ ${ }^{2}$ Maxar Technologies, Palo Alto, California, USA
}

\begin{abstract}
A droplet hitting a thin liquid layer can cause different phenomena such as bouncing, splashing, fingering, etc. In this paper, these interactions are studied experimentally when a droplet impacts on different liquid film thicknesses that causes different impact regions. In the experiments, a colored water droplet is impinged on liquid films from different heights to create Weber numbers between 121 and 304. Also, different liquid film thicknesses are prepared to create various dimensionless film thickness $h^{*}$ between 0.4 to $1.6 \mathrm{~mm}$. All of these processes are recorded with a color high-speed camera at a recoding rate of $2000 \mathrm{fps}$. The color contrast between the droplet and the colorless liquid film created a distinction in images and improved observation of details. The color contrast is especially used to analyze the finger formation. This process is studied both during the crown formation and after the crown disappears. As a novel contribution, a different type of fingering mechanism is identified. We number is found to be the main contributor of this finger formation. On the other hand, the dimensionless film thickness $h^{*}$ is mainly responsible for the formation of these fingers whether they will appear above or inside the crown.
\end{abstract}

\section{Keywords}

Droplet impact, liquid film, fingering, mixing, high speed imaging

\section{Introduction}

Droplet impact on dry and wet surfaces has many traditional and emerging applications, such as inkjet printing, spray cooling and quenching, liquid jet cleaning, and internal combustion engines [1]. These droplet-based methods are also used to deposit polymers to create electronic circuits, construct 3-D parts out of wax or other materials in new additive manufacturing methods. Moreover, these methods are linked to computer-controlled droplet generators which increase the accuracy of the product [2]. Micro droplet deposition is also another modern technology that has broad usage for microelectronics, including biomedical engineering [3]. A recent study shows that drop impact printing is now widely accessible as it is significantly simple and cost-effective [4]. As these processes are centered around droplet impact behavior, studying the droplet impact dynamics is a necessity. The attention given to this field is increased considering the importance of droplet impacts in heat and mass transfer processes, especially with the rapid growth of highspeed imaging and high precision measurement techniques. The number of articles associated with droplet impacts has multiplied by a factor of 10 over the last 50 years [5].

When a droplet impinges on a dry surface, the droplet may experience air bubble entrapment, it may spread smoothly and deposit. However, when the impact velocities are high, it may create corona splash, recedes if the droplet has overspread, or rebounds if the substrate is hydrophobic. Moreover, both outward and inward waves are present in all types of impacts. The spreading factor $(\beta)$, which is defined as the ratio of the expanding droplet diameter to the original droplet 
diameter, versus dimensionless time is used to explain the spreading behavior of the droplet. In line with spreading factor, there are four levels of the impact: kinematic stage, scattering stage, relaxing stage, and wetting stage [6]. While the contact angle can influence spreading at the very beginning of the impact [7], these stages are generally regulated by $R e$ and We numbers with high inertial forces. The substrate role becomes more important at the end of the impact. Although a wetting surface can lead to significant additional expansion, a partly wetting surface with low surface energy can lead to a substantial decrease in expansion of the rim [8]. Moreover, on hydrophobic surfaces, which is a non-wetting surface, huge amount of receding might occur. In some cases, even rebounding can be observed.

In thin liquid films, splashing due to crown formation can be correlated with We number [9]. When the deep liquids are the case, gravity effect becomes significant, so Froude $(F r)$ and Bond $(B)$ numbers are relevant. Here, the dimensionless thickness $h^{*}$ has no visible effect. Wang and Chen [10] performed thin liquid film experiments, accounting for dimensionless film thickness $h{ }^{*}<0.1$. They noticed a complete breakdown of the crown with no central jet in such thin films. They noted that in this regime, the splashing threshold was strongly sensitive to surface characteristics, and the importance of film thickness was reduced. Cossali et al. [11] studied the time evolution of various droplet impact characteristics, such as crown height, diameter, and thickness, secondary droplet diameters, and the number of fingers formed on the crown. These parameters were found not to be strictly linked to $h^{*}$, but some other parameters, such as crown height, depend on the We number.

Many researchers have recently studied different droplet impact scenarios out of curiosity or due to current applications. For instance, a recent study shows that the bubble entrapment can be detected by using an acoustic sensor, of which the frequency of vibration signal is inversely proportional to the droplet size. Moreover, MEMS-based acoustic sensors are employed to detect and measure the diameter of the droplet [12]. In another study, it has been shown that droplet effect on a low-viscosity, low-surface-tension liquid film induces surface tension concentration gradient on the crown wall and results in breakup. Consequently, a fascinating shower of droplets was observed shattering from the wall of the crown [13]. In another work, the effect of fluid and surface characteristics on the outcome and transition between each regime of droplet impact for $h^{*}<1$ was explored [14]. By raising the liquid viscosity, the production of secondary droplets was reduced, and the time scale of the impact phenomena was decreased by lowering the surface tension. It is worth mentioning that having many secondary droplets might be undesirable in some cases. Therefore, to reduce them, adding a small amount of a vesicular surfactant is found to be very efficient on hydrophobic surfaces. The vesicular surfactant limits the motion of liquid by altering the wettability transition, thus it restricts the formation of the secondary droplets [15].

Although there are numerous studies on rapid impact phase of a droplet, there is no work, to the best of our knowledge, to treat the incoming droplet independently. Thus, in this work, after the impact, the behavior and evolution of the incoming droplet and the liquid film are studied altogether with the data published in previous works [18]. 


\section{Methods}

The experimental setup shown in Figure 1 was used to analyze the effect of a droplet impinging on an existing thin liquid film with a known thickness. The primary objective of this work was to demonstrate the impact dynamics of a droplet inside a liquid surface. Therefore, a different colored droplet was used to visualize the dynamics of an incoming droplet from the film. It is made possible by the combination of deionized water and food dye. Methylene blue (MACKLIN) was used to produce blue color in the impinging droplet.

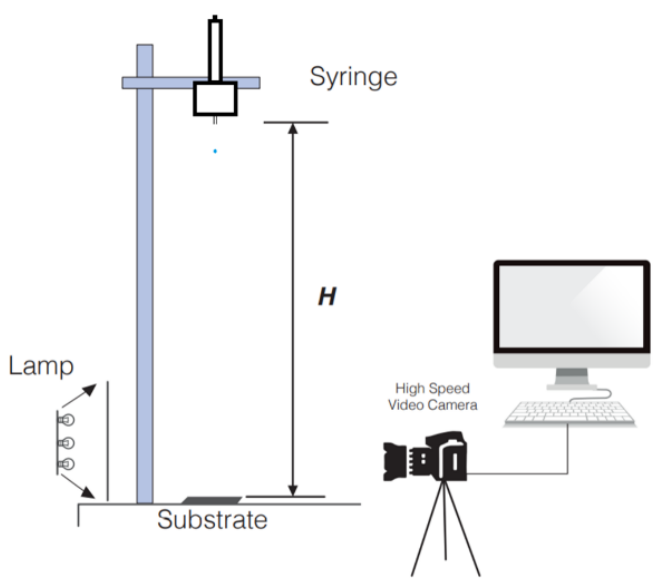

Figure 1: The Experimental Setup

The atmospheric pressure was maintained at $1 \mathrm{~atm}$ throughout the experiment. The test liquids and the temperature of the laboratory were held at $25^{\circ} \mathrm{C}$. The surface tension coefficients of the test liquids for the water and blue water were very close, namely $0.07258 \mathrm{~N} / \mathrm{m}$ and $0.07042 \mathrm{~N} / \mathrm{m}$, respectively. The droplets were produced by using a syringe (HAMILTON 1001 TPLT), which in all situations, produces the same droplet size. Image analysis results showed that the droplet diameter was $4.14 \mathrm{~mm}$. The syringe was hold firmly by a clamp and adjustable stand with $1 \mathrm{~mm}$ resolution and $500 \mathrm{~mm}$ movement range. In the experiments, $50 \mathrm{~mm} \times 50 \mathrm{~mm}$ fluorine-doped tin oxide (FTO) coated glass was used as the substrate. The substrates were ultrasonically washed and cleaned for every case. The substrate surface was covered by deionized water to form a liquid film. Here, to find the thickness of the film, a precision scale was used under the substrate. So, the thickness of the liquid film was determined by weight of the added liquid to the substrate as liquid thin film. Simply, the mass of liquid added to the substrate (known) is equal to the product of area of the substrate (known), thickness of the film (unknown) and density of the liquid (known).

The video capturing system includes a high-speed video camera (Photron, FASTCAM-SA3, Japan) and a Nikkor 105-mm micro lens (NIKON, Japan). The process was recorded by the highspeed video camera at a recording speed of 2000 frames per second (fps) and $1024 \times 1024-p i x e l$ resolution. With a high-intensity LED lamp, flicker-less backlighting was produced. High speed video camera has its own software which is called Photron Fastcam Viewer. The software allows capturing, recording, and storing the droplet impact process.

In these experiments, the variables are the droplet release height and liquid film thickness: 4 different droplet heights including 10,15,20, and $25 \mathrm{~cm}$ and 4 different liquid film thicknesses 
including 400, 800, 1200, and $1600 \mu \mathrm{m}$. Each experimental case was performed 5 times to test the repeatability. In the experiments, the droplet was released from the syringe, with an initial velocity of zero. After the detachment from the syringe, the droplet was then accelerated by gravity. The impact velocity $V$ was measured by image analysis. By varying the impact height $\mathrm{H}$, the velocity, $V$ is controlled. 4 different heights created 4 different impact velocities including 1.40, $1.72,1.98,2.21 \mathrm{~m} / \mathrm{s}$. Dimensionless film thickness $h^{*}$ is found by $h^{*}=h / D, h$ being the film thickness and $D$ the initial droplet diameter. 4 different liquid film thicknesses created 4 different $\mathrm{h}^{*}$ including $0.092,0.183,0.275,0.367$. To have a better understanding of the rapid impact procedure, images during the crown formation stage were collected, compared, and examined.

\section{Results and Discussion}

Figure 2 shows the effect of $h^{*}$ on droplet impact, discussed in detail in ref. [18]. Here the focus is on another phenomenon, namely, the instability and fingering of the impinged droplet inside the liquid film. The theory behind the finger formation is based on Rayleigh-Taylor type instability on the drop circumference during the expansion phase of the droplet. The radial portion of the droplet is slowed down by the friction forces and the pressure difference between the surrounding fluid (a gas on solid surface impact and a liquid on wet surface impact) results in larger instability at this point. Here, during the kinematic and spreading process of the impact, the axisymmetric trends of droplet development are often disrupted by azimuthal disturbances. The growth of these disturbances results in fingering at the surface of a spreading droplet.

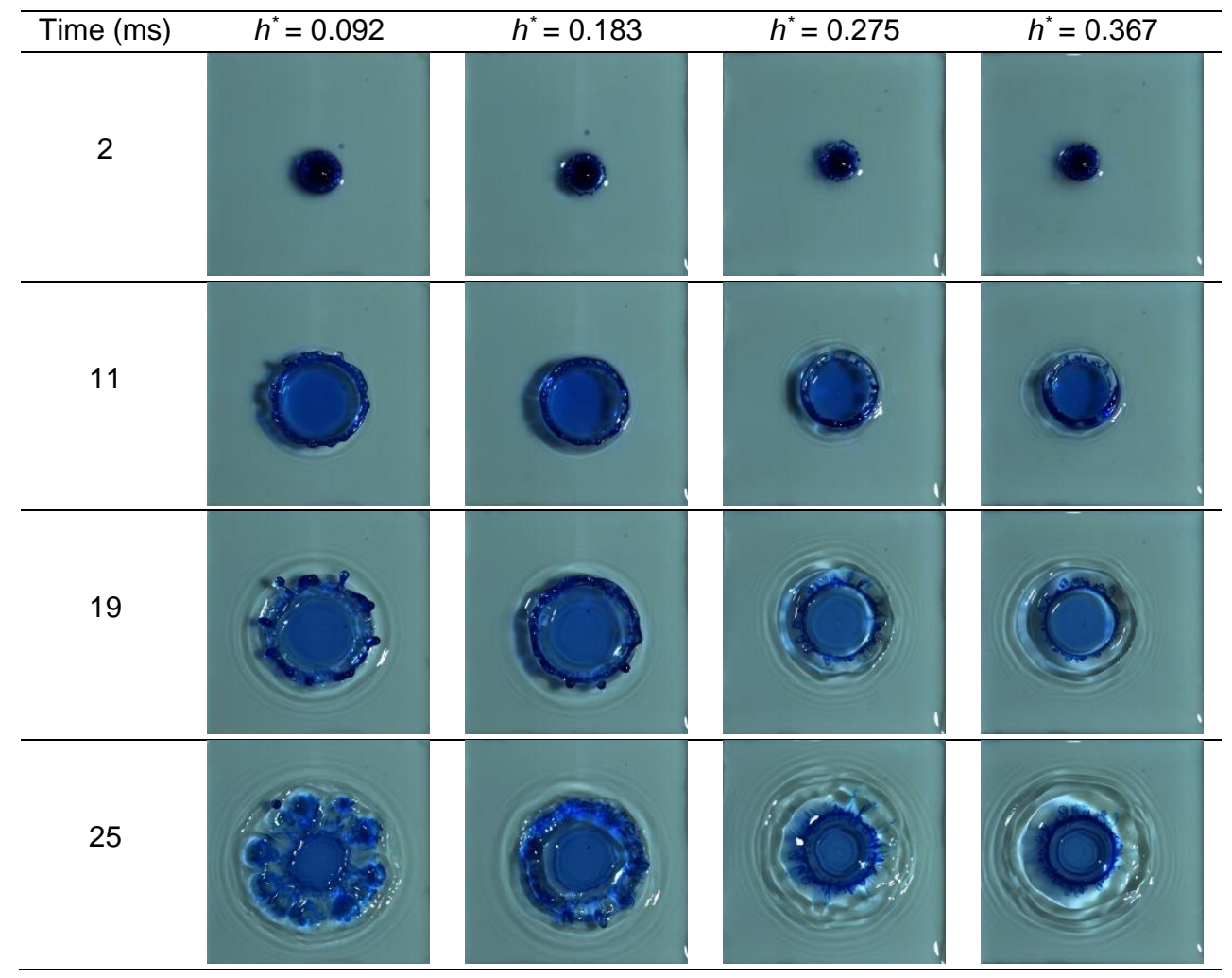

Figure 2: Droplet impact phenomena at $W e=182$. Images are $5 \mathrm{~cm} \times 5 \mathrm{~cm}$. Some of the images were originally published in [18]. 
For the case of dry surface impact, wettability is one of the key variables. The structure of droplet impact creates a spatial gradient on the surface that causes directional asymmetry which eventually contributes to an unstable rim. Larger contact angle situations result in faster development of the unstable rim. In the wet impact cases, although the roughness of the surface is at the wheel, it loses its effect as liquid film thickness increases.

Single droplet impacts on liquid films are divided into four regions: thin liquid film, liquid film, shallow pool, and deep pool [17]. In the present study, two of these regions, thin liquid film and liquid film, are used and compared in the experiments. Depending on the wet film thickness to droplet size ratio $\left(h^{*}\right)$, one can observe different phenomena clearly. The most obvious one is the ability to produce fingers and secondary droplets during the crown formation phase. While thin liquid films promote fingering, liquid films make this formation difficult due to a larger $h^{*}$ value. However, using the contrast between the droplet and the wet film, a different kind of fingering mechanism can be observed in all liquid film regions. As shown in Figure 3a, the fingers are produced but encapsulated by the crown rim in the liquid film region. The blue-colored area depicts a regular fingering formation. This formation can be explained as follows. It is known that these types of formations are present due to Rayleigh-Taylor instability. In thin-film regions, the droplet kinetic energy helps overcome the liquid barrier and results in liquid-gas interaction. Due to the high density and viscosity difference between the liquid and air, the expanding crown rim starts to accumulate at certain positions, and this produces fingers and secondary droplets [4].

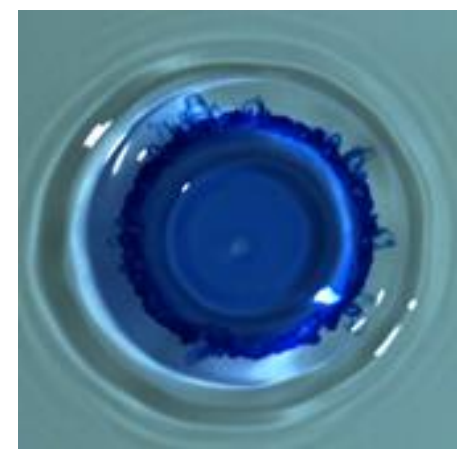

(a)

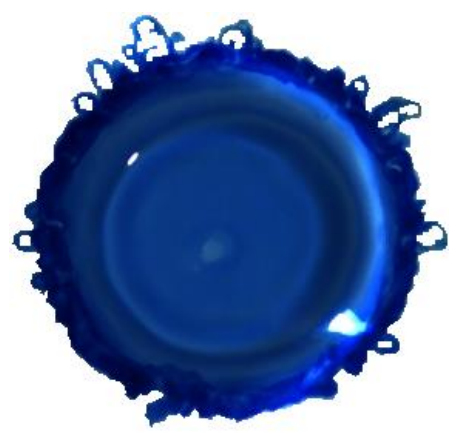

(b)

Figure 3: (a) Droplet impact phenomena at $W e=304, h^{*}=0.367$ and $t=20 \mathrm{~ms}$. The image is $3.2 \mathrm{~cm} \times 3.2 \mathrm{~cm}$.

(b) Extracted image of the same droplet inside the liquid film. Mean diameter of the image is $1.8 \mathrm{~cm}$.

In the case of liquid films, these types of formations were not observed due to the device limitations in the past. In the experiments, thanks to the color difference between the thin liquid film and the droplet, it was possible to observe the independent behavior of the incoming droplet after it merges with liquid film. Although small $h^{*}$ cases do not show a remarkable difference, high $h^{*}$ cases revealed that the incoming droplet experiences a different type of expansion inside the thick crown rim alongside the common expansion behavior. Figure 3a shows an instant during the late stage of an expanding droplet on the liquid film. If the incoming droplet was the same kind of the liquid film (same color), one would see an expanding crown with a thick rim without any trace of finger formation. However, Figure $3 \mathrm{~b}$ shows the behavior of the incoming droplet alone, inside the liquid film. A more interesting point of view appears when this instance is compared 
with an expanding droplet on a solid surface as shown in Figure 4. Although clear differences are present, the resemblance is uncanny. From these images, it can be intuitively concluded that the impinging droplet on a liquid film experiences an expanding behavior different from its joint behavior with the liquid film.

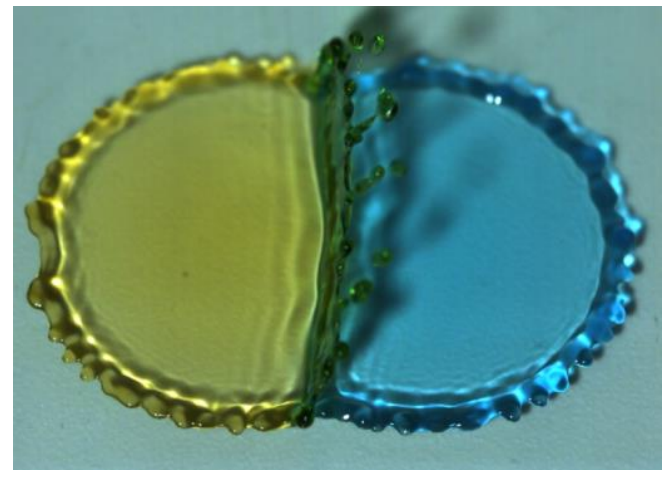

Figure 4: Two simultaneous droplet Impact on a dry surface. The image is $3 \mathrm{~cm} \times 2.2 \mathrm{~cm}$. More detail can be found in ref. [19]

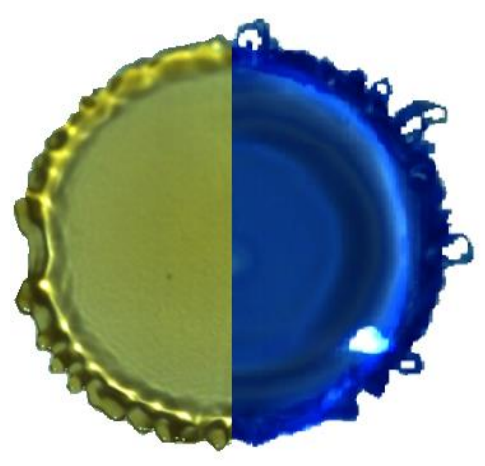

(a)

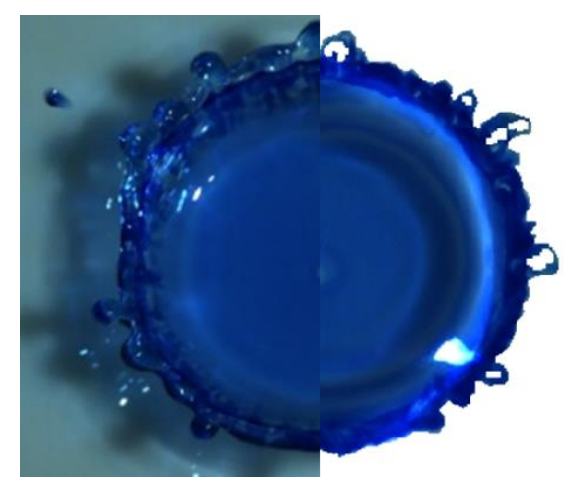

(b)

Figure 5: (a) Dry surface impacts (left half) vs. in-liquid fingering (right half). (b) Crown fingering in thin film impact (left half) vs. in-liquid fingering (right half). Mean diameter of the images are $1.8 \mathrm{~cm}$.

The splashing is influenced by the density, followed by the viscosity, and last by the mean free path of the surrounding gas [16]. When the thin liquid film is used as the surrounding fluid, it is obvious that it will produce similar behavior to the liquid-gas interaction since both experience fluid-fluid interaction. The shape of the in-liquid fingering with a dry surface impact in Figure $5 a$ is compared with the fingering in the crown during thin liquid impact in Figure 5b. In the expansion phase of the droplet, when the kinetic energy of the expanding droplet decreases enough, it starts to accumulate as a rim on the outer surface. This means that the expansion energy of the droplet is no longer strong enough to surpass viscous forces to push the air outside. In the case of inliquid fingering, same conclusion can be drawn that a similar rim is produced, this time due to the surrounding liquid. Moreover, as Figure 5b exhibits, since the effect of surface forces is decreased due to the thin liquid film, a larger amount of the energy is used to push the liquid outside and local instabilities are produced, which results in finger formation. One can observe similar inner fingers during the in-liquid fingering. 
Another evidence to these in-liquid fingering is the fingering-like formation produced after the crown disappears. For example, in Figure 6c the droplet impact for $W e=182$ and $h^{*}=0.367$ does not show any fingering formation, only a thick crown rim is present. However, the instance after the crown formation supports the existence of these in-liquid fingers as it is shown in Figure $6 \mathrm{~d}$. According to the six observed mixing mechanisms of miscible liquids during droplet impact on a liquid film [18], the second and third mixing mechanisms are present for the inner crown formations. The two mixing mechanisms, which are crown with acute wall angle that spreads over the thin film and crown fingering, can be observed in Figure $6 \mathrm{a}$ and $6 \mathrm{~b}$ as well. Here the main difference is the velocity gradient of the regular finger is a lot bigger than the in-liquid fingering due to its contact with air. On the other hand, the inner finger is exposed into much higher density and viscosity, so its outer movement will be much more limited as it can be observed in Figure 6c and $6 \mathrm{~d}$. According to the observations from experiments, Weber number is found to be the main contributor of the finger formation, either a regular finder or in-liquid finger. On the other hand, the dimensionless film thickness $h^{*}$ is mainly responsible whether these fingers will appear above or inside the crown.

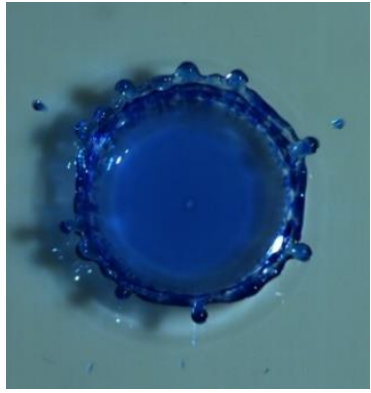

(a)

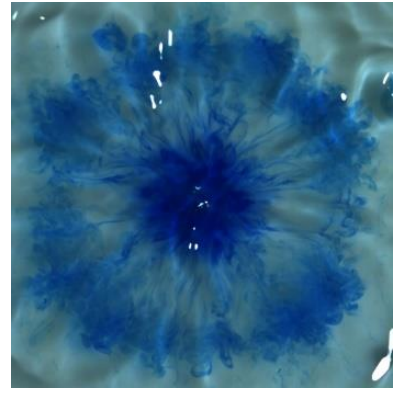

(b)

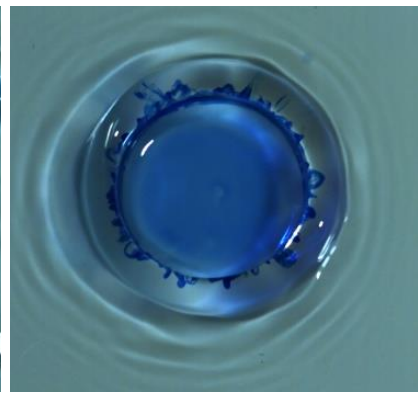

(c)

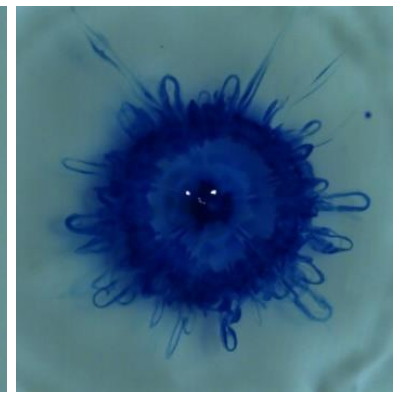

(d)

Figure 6: (a) and (b) represent the traditional fingering mechanism for We $=182$ and $h^{*}=0.092$ during and after the crown formation, respectively. (c) and (d) represent the in-liquid fingering mechanism for $W e=182$ and $h^{\star}=0.367$ during and after the crown formation, respectively. Images are $5 \mathrm{~cm} \times 5 \mathrm{~cm}$.

\section{Conclusion}

In this work, droplet impact of a dyed water droplets onto clear water films was studied using a colored high-speed camera. Clear evocatory and complex formations inside the liquid film are shown. It was observed that in liquid film regions, the incoming droplet behavior inside the liquid film resembles droplet impact on dry surfaces and thin liquid films. As a novel contribution, a new type of fingering mechanism is presented in liquid film regions.

\section{References}

[1] Eslamian, M., 2014, Spray-on thin film PV solar cells: advances, potentials and challenges. Coatings, 4(1), 60-84

[2] Dalili, A., Chandra, S., Mostaghimi, J., Fan, H. C., \& Simmer, J. C., 2014, Formation of liquid sheets by deposition of droplets on a surface, Journal of colloid and interface science, 418, 292299.

[3] Thompson, A. B., Tipton, C. R., Juel, A., Hazel, A. L., \& Dowling, M., 2014, Sequential deposition of overlapping droplets to form a liquid line, Journal of Fluid mechanics, 761, 261-281. 
[4] Modak, C. D., Kumar, A., Tripathy, A., \& Sen, P., 2020, Drop impact printing, Nature communications, 11(1), 1-11.

[5] Brutin, D., \& Starov, V., 2018, Recent advances in droplet wetting and evaporation, Chemical Society Reviews, 47(2), 558-585.

[6] Rioboo, R., Marengo, M., \& Tropea, C., 2002, Time evolution of liquid drop impact onto solid, dry surfaces, Experiments in fluids, 33(1), 112-124.

[7] Xu, Y., Vincent, S., He, Q. C., \& Le-Quang, H., 2019, Spread and recoil of liquid droplets impacting on solid surfaces with various wetting properties, Surface and Coatings Technology, 357, 140-152.

[8] Soltani-Kordshuli, F., \& Eslamian, M., 2017, Impact dynamics and deposition of pristine and graphene-doped PEDOT: PSS polymeric droplets on stationary and vibrating substrates, Experimental Thermal and Fluid Science, 89, 238-248.

[9] Macklin, W. C., \& Metaxas, G. J., 1976, Splashing of drops on liquid layers, Journal of applied physics, 47(9), 3963-3970.

[10] Wang, A. B., \& Chen, C. C., 2000, Splashing impact of a single drop onto very thin liquid films, Physics of fluids, 12(9), 2155-2158.

[11] Cossali, G. E., Marengo, M., Coghe, A., \& Zhdanov, S., 2004, The role of time in single drop splash on thin film, Experiments in Fluids, 36(6), 888-900.

[12] Nguyen, T. V., \& Ichiki, M., 2020, Bubble entrapment during the recoil of an impacting droplet, Microsystems \& Nanoengineering, 6(1), 1-7.

[13] Thoroddsen, S. T., Etoh, T. G., \& Takehara, K., 2006, Crown breakup by Marangoni instability, Journal of Fluid Mechanics, 557, 63-72.

[14] Pan, K. L., \& Hung, C. Y., 2010, Droplet impact upon a wet surface with varied fluid and surface properties, Journal of colloid and interface science, 352(1), 186-193.

[15] Song, M., Ju, J., Luo, S., Han, Y., Dong, Z., Wang, Y., ... \& Jiang, L., 2017, Controlling liquid splash on superhydrophobic surfaces by a vesicle surfactant, Science advances, 3(3).

[16] Burzynski, D. A., \& Bansmer, S. E., 2019, Role of surrounding gas in the outcome of droplet splashing, Physical Review Fluids, 4(7).

[17] Ersoy, N. E., \& Eslamian, M., 2020, Phenomenological study and comparison of droplet impact dynamics on a dry surface, thin liquid film, liquid film and shallow pool, Experimental Thermal and Fluid Science, 112.

[18] Ersoy, N. E., \& Eslamian, M., 2019, Capillary surface wave formation and mixing of miscible liquids during droplet impact onto a liquid film. Physics of Fluids, 31(1).

[19] Ersoy, N. E., \& Eslamian, M., 2020, Central uprising sheet in simultaneous and nearsimultaneous impact of two high kinetic energy droplets onto dry surface and thin liquid film. Physics of Fluids, 32(1). 